Pacific

Journal of

Mathematics

ALL LINKS ARE SUBLINKS OF ARITHMETIC LINKS

MARK D. BAKER 


\title{
ALL LINKS ARE SUBLINKS OF ARITHMETIC LINKS
}

\author{
MARK D. BAKER
}

\author{
We show that every link in $S^{3}$ is a sublink of an arithmetic \\ link.
}

\section{Introduction.}

In this paper we show that arithmetic links play a central role in the Dehn surgery description of closed 3-manifolds. Let $L \subset S^{3}$ be a link of (one or more) circles. We prove that $L$ is a sublink of an arithmetic link. Specifically:

Theorem 1. Let $L \subset S^{3}$ be a link. Then $L$ is a sublink of a link $J$ such that $S^{3} \backslash J$ is homeomorphic to $\mathbb{H}^{3} / \Gamma$, where $\Gamma$ is a torsion-free subgroup of finite index in the Bianchi group $P S L_{2}(\mathbb{Z}[i])$.

Since every closed, orientable 3-manifold can be obtained by Dehn surgery on a link in $S^{3}$ (see $[\mathbf{L i}]$ ), we have:

Theorem 2. Every closed, orientable 3-manifold can be obtained by Dehn surgery on an arithmetic link in $S^{3}$.

While it is known that every closed orientable 3-manifold $M$ contains an arithmetic link $L$ (since the figure-eight knot complement is both arithmetic and universal), Theorem 2 asserts that $L$ can be chosen so that $M \backslash L$ is homeomorphic to the complement of a link in $S^{3}$.

Recall that a link $L$ in $S^{3}$ (resp. in $M$ ) is hyperbolic if $S^{3} \backslash L$ (resp. $M \backslash L$ ) is homeomorphic to $\mathbb{H}^{3} / \Gamma$, where $\mathbb{H}^{3}$ is hyperbolic 3 -space and $\Gamma$ a discrete, torsion-free, finite covolume subgroup of $P S L_{2}(\mathbb{C})$. We say that $L$ is arithmetic if $\Gamma$ can be chosen commensurable with a Bianchi group $P S L_{2}\left(\mathcal{O}_{m}\right)$, where $\mathcal{O}_{m}$ is the integers of the imaginary quadratic number field $\mathbb{Q}(\sqrt{-m})$ (see $[\mathbf{R}]$ for a more general discussion). Finally, $L$ is a sublink of $J$ if it is a union of components of $J$. 


\section{Proof of Theorem 1.}

Denote by $L_{1}$ the 6 -circle link in Figure 1.

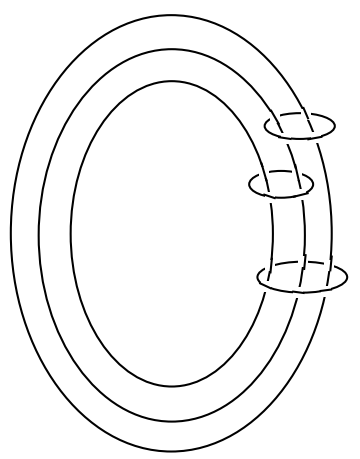

Figure 1.

We prove our result by showing that:

i) $L_{1}$ is an arithmetic link: $S^{3} \backslash L_{1} \cong \mathbb{H}^{3} / \Gamma_{1}$, where $\Gamma_{1}$ is a torsion-free subgroup of the Bianchi group $P S L_{2}(\mathbb{Z}[i])$.

ii) Every link $L$ occurs as a sublink of a link $J$ such that $S^{3} \backslash J$ is a covering space of $S^{3} \backslash L_{1}$.

Thus $S^{3} \backslash J \cong \mathbb{H}^{3} / \Gamma$, where $\Gamma \subset \Gamma_{1} \subset P S L_{2}(\mathbb{Z}[i])$, and so $J$ is arithmetic and contains $L$ as a sublink. We prove the arithmeticity of $L_{1}$ in 2.1. Section 2.2 is devoted to proving property ii).

2.1. . The link $L_{1}$ is arithmetic since $S^{3} \backslash L_{1}$ is a 2-fold cover of $S^{3} \backslash L_{0}$ where $L_{0}$ is the four component arithmetic link in Figure 2.

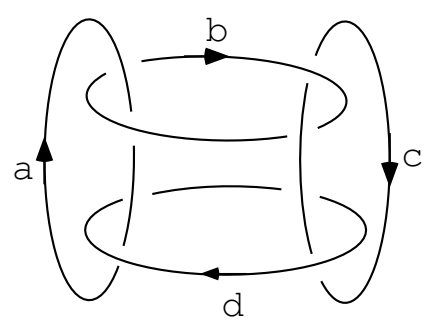

Figure 2.

Indeed, $S^{3} \backslash L_{0} \cong \mathbb{H}^{3} / \Gamma_{0}$ where

$$
\Gamma_{0}=\left\langle\left[\begin{array}{ll}
1 & 2 \\
0 & 1
\end{array}\right],\left[\begin{array}{cc}
1 & 2 i \\
0 & 1
\end{array}\right],\left[\begin{array}{cc}
1 & 0 \\
1-i & 1
\end{array}\right],\left[\begin{array}{cc}
1 & 0 \\
1+i & 1
\end{array}\right]\right\rangle \subset P S L_{2}(\mathbb{Z}[i])
$$


(see [Wi] Example 3 for a detailed treatment), and $S^{3} \backslash L_{1}$ is the 2-fold cover corresponding to the kernel of the map $\theta: \pi_{1}\left(S^{3} \backslash L_{0}\right) \rightarrow \mathbb{Z} / 2 \mathbb{Z}$ given by $\theta(a)=\theta(c)=1$ and $\theta(b)=\theta(d)=0$.

A second proof of the arithmeticity of $L_{1}$ goes as follows (see $\left.[\mathbf{R}]\right): \Gamma_{1}$ is a subgroup of $P S L_{2}(\mathbb{Z}[i])$ if and only if $\operatorname{tr}\left(\Gamma_{1}\right)=\left\{\operatorname{tr}(\gamma) \mid \gamma \in \Gamma_{1}\right\} \subset \mathbb{Z}[i]$, which is true if and only if, for a set of generators $\gamma_{1}, \ldots, \gamma_{n}$ of $\Gamma_{1}$, the following traces are in $\mathbb{Z}[i]: \operatorname{tr}\left(\gamma_{i}\right)$ and $\operatorname{tr}\left(\gamma_{i} \gamma_{j}\right), i<j$. We used SnapPea $([\mathbf{W}])$ to compute a matrix representation for $\pi_{1}\left(S^{3} \backslash L_{1}\right)$ and verify that the above traces are indeed in $\mathbb{Z}[i]$.

2.2. . By the Alexander braiding theorem (see $[\mathbf{B}-\mathbf{Z}]$ ) any link can be realized as the closure of an $n$-braid (Figure 3 ).

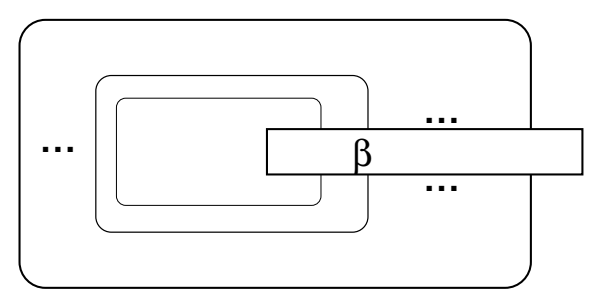

\section{Figure 3.}

Here $\beta=\alpha_{i_{k}}^{s_{k}} \cdots \alpha_{i_{1}}^{s_{1}}$ is a product of powers of the standard generators of the braid group $B_{n}$ (Figure 4 ).

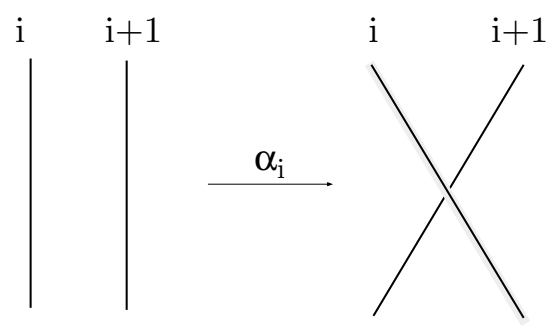

Figure 4.

We show that $L$ is a sublink of a $J$ such that $S^{3} \backslash J$ is a cover of $S^{3} \backslash L_{1}$ hence arithmetic. Before giving the construction of $J$ in the general case, we first illustrate the process by treating the case when $L$ is the trefoil knot. 
2.2.1. . The trefoil knot is the closure of the 2 -strand braid $\beta=\alpha^{3}$ (Figure 5$)$.

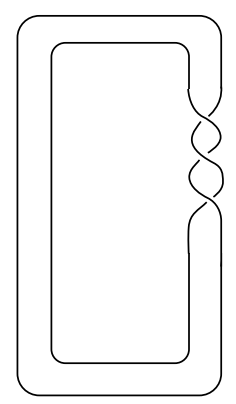

Figure 5 .

Let $X^{1}$ be the 2-fold cover of $X \cong S^{3} \backslash L_{1}$ branched over the circle $c$ (see Figure 6. We draw only the braid part of the vertical components in order to save space). This cover is again a link complement since we are branching over an unknotted component of $L_{1}$.
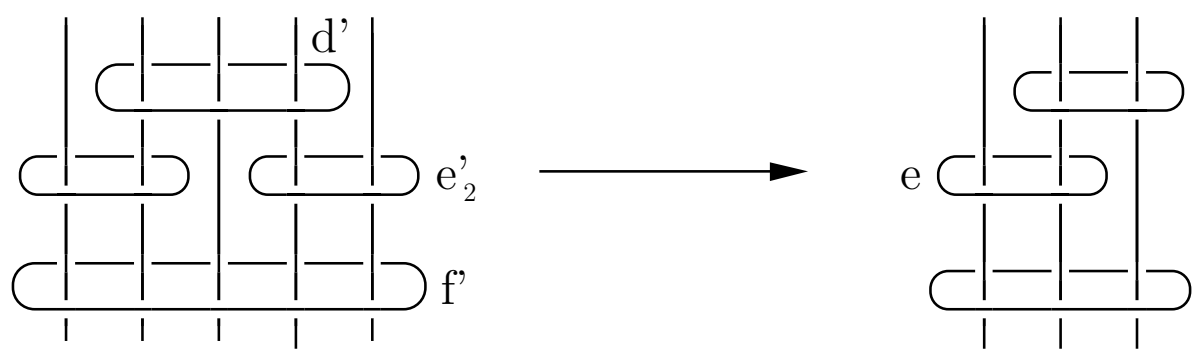

Figure 6.

Now we transform $X^{1}$ into $S^{3} \backslash J$ by performing a $3 / 2$ twist about the circle $d^{\prime}$ i.e., cutting along the disk $D^{\prime}$ bounded by $d^{\prime}$, twisting through $3 \pi$ and regluing. This has the effect of $\alpha^{3}$ on the circles $b_{1}^{\prime}, b_{2}^{\prime}$, changing them into the desired trefoil knot (Figure 7). The key point here is that $S^{3} \backslash J$ is also a 2-fold cover of $S^{3} \backslash L_{1}$. We now examine this point in greater detail. 
2.2.2. . We show (with notation as above) that any $n / 2$ twist about $d^{\prime}$ transforms $X^{1}$ into another 2-fold cover of $X$. Since integer twists about $d^{\prime}$ are homeomorphisms, it suffices to consider the case of a $1 / 2$ twist. Note that $D^{\prime} 2$-fold covers $D$, a disk bounded by circle $d$ in $X$ (Figure 8 ). Since the complement of an unknotted circle in $S^{3}$ is a solid torus, cutting along $D^{\prime}$ and $D$ transforms $X^{1}$ and $X$ into solid cylinders (minus circles and arcs): $Y^{1} 2$-fold covering $Y$. Now glue the two copies of $D$ by the identity to get $X$, and note that there are two gluings of the $D^{\prime \prime}$ s that give a cover of $X$ : The identity which gives back $X^{1}$ and the order 2 automorphism of $D^{\prime}$ which yields the cover corresponding to the $1 / 2$ twist mentioned above.
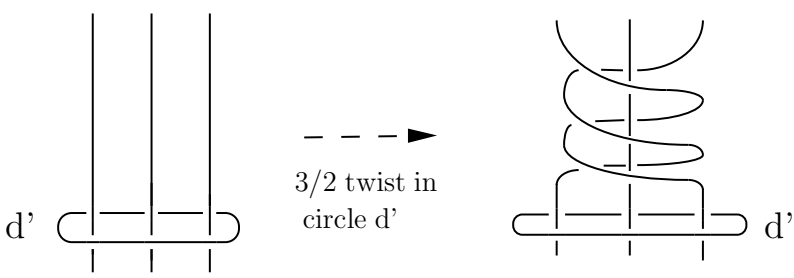

Figure 7.
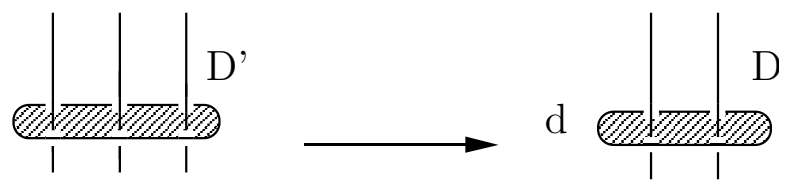

Figure 8.

2.2.3. . Given $L$ the closed $n$-braid corresponding to $\beta=\alpha_{i_{k}}^{s_{k}} \cdots \alpha_{i_{1}}^{s_{1}}$, we obtain $J$ by the construction in steps $1-4$ below.

1) Let $X^{1}$ be the 2 -fold cover of $X \cong S^{3} \backslash L_{1}$ branched over the circle $c$.

2) For $r>1$, let $X^{r}$ be the 2-fold cover of $X^{r-1}$ branched over the rightmost preimage of the circle $a$.

The cover $X^{r}$ is a link complement (since branched over an unknotted circle in $X^{r-1}$ ) containing $2^{r}$ unknotted, unlinked preimages of the circle $b$. Choose $r$ so that $2^{r}>n$.

3) Let $\widetilde{X}$ be the $k$-fold cyclic cover of $X^{r}$ branched over the preimage of circle $f$ (see Figure 9). 
4) Transform $\tilde{X}$ into $S^{3} \backslash J$ by twists corresponding to $\beta=\alpha_{i_{k}}^{s_{k}} \cdots \alpha_{i_{1}}^{s_{1}}$ in the appropriate preimages of circles $d$ and $e$.
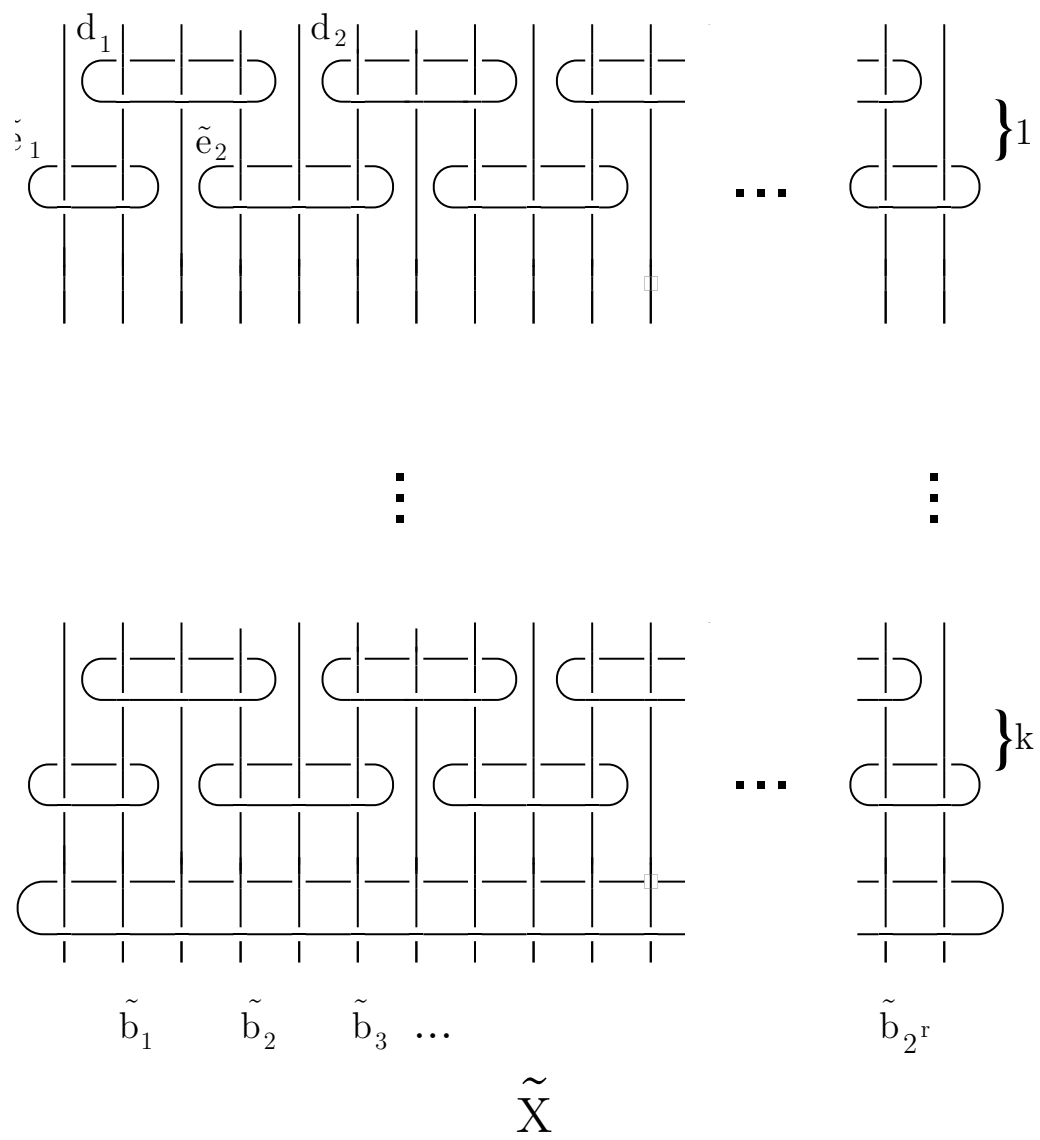

\section{Figure 9.}

Consider the $n$ left-most preimages $\widetilde{b}_{1}, \ldots, \widetilde{b}_{n}$ in $\widetilde{X}$ of circle $b$. Adjacent circles are linked by preimages of $d$ and $e: \widetilde{b}_{1}, \widetilde{b}_{2}$ by $\widetilde{d}_{1} ; \widetilde{b}_{2}, \widetilde{b}_{3}$ by $\widetilde{e}_{2}$ and so forth. This pattern is repeated in $k$ blocks from top to bottom in Figure 9. Now, for each of the $k$ factors $\alpha_{i_{j}}^{s_{j}}$ in $\beta$ perform a $s_{j} / 2$ twist in the $\widetilde{d}$ or $\widetilde{e}$ linking $\widetilde{b}_{i_{j}}, \widetilde{b}_{i_{j}+1}$ in the $j$-th block. This changes the circles $\widetilde{b}_{1}, \ldots, \widetilde{b}_{n}$ into $L$. Finally, $S^{3} \backslash J$ is a $\left(2^{r} k\right)$-fold cover of $S^{3} \backslash L_{1}$ as explained in 2.2.2.

Acknowledgements. This paper grew out of questions put to me by D. Long. I also thank J. Hubbard for help in getting started with TEX. 


\section{References}

[B-Z] G. Burde and H. Zieschang, Knots, deGruyter Studies in Mathematics, 5, W. deGruyter, Berlin, New York, 1985, MR 87b:57004, Zbl 0568.57001.

[Li] W.B.R. Lickorish, A representation of orientable combinatorial 3-manifolds, Annals of Math., 76 (1962), 531-538, MR 27 \#1929, Zbl 0106.37102.

[R] A. Reid, Arithmeticity of knot complements, J. Lond. Math. Soc.(2), 43 (1991), 171-184, MR 92a:57011, Zbl 0847.57013.

[W] J. Weeks, SnapPea 2.4 PPC, Available from http://www.northnet.org/weeks.

[Wi] N. Wielenberg, The structure of certain subgroups of the Picard group, Math. Proc. Camb. Phil. Soc., 84 (1978), 427-436, MR 80b:57010, Zbl 0399.57005.

Received June 27, 2000 and revised February 14, 2001.

IRMAR

UNIVERSITÉ DE RENNES 1

35042 RENNES CEDEX

FRANCE

E-mail address: baker@univ-rennes1.fr 